\title{
Manifestations buccales chez les enfants et adolescents infectes par le VIH à Kinshasa (RDC) et facteurs associés
}

\section{Oral manifestations among HIV infected children and adolescents in Kinshasa (DRC) and associated factors}

\author{
Bakana L ${ }^{1}$, Dilu $\mathrm{F}^{2}$,Lelo $\mathrm{P}^{3}$,Kowe $\mathrm{N}^{1}$, Bakambana $\mathrm{G}^{4}$,Songo $\mathrm{F}^{1}$, Kitetele $\mathrm{F}^{3}$, Dilu $\mathrm{J}^{2}$, Ahuka $\mathrm{S}^{5,6}$, Bolenge $\mathrm{J}^{1}$, \\ Situakibanza $\mathrm{H}^{7}$ \\ ${ }^{1}$ Département d'Odontostomatologie, Cliniques Universitaires de Kinshasa, RD Congo. \\ ${ }^{2}$ Département de Chirurgie, Cliniques Universitaires de Kinshasa, RD Congo. \\ ${ }^{3}$ Service de Maladies Infectieuses, Hôpital Pédiatrique de Kalembe-Lembe, Kinshasa, RD Congo. \\ ${ }^{4}$ Service de Dentisterie, Hôpital Pédiatrique de Kimbondo, RD Congo. \\ ${ }^{5}$ Service de Microbiologie, Cliniques Universitaires de Kinshasa, RD Congo. \\ ${ }^{6}$ Institut National de Recherche Biomédicale (INRB/Kinshasa), Kinshasa, RD Congo. \\ ${ }^{7}$ Département de Médecine Interne, Cliniques Universitaires de Kinshasa, RD Congo.
}

\begin{abstract}
Auteur correspondant : Pr Hippolyte Situakibanza Nani-Tuma, Département de Médecine Interne,
Département de Médecine Tropicale, Maladies Infectieuses et Parasitaires, Faculté de Médecine, Université de Kinshasa E-mail : hsitua@gmail.com
\end{abstract}

\section{RESUME}

Objectifs : Mesurer l'ampleur des manifestations buccales chez les enfants et adolescents VIH positif fréquentant le milieu hospitalier de Kinshasa et en identifier les facteurs associés. Méthodes : Il s'est agi d'une étude transversale analytique, conduite dans deux hôpitaux pédiatriques de Kinshasa (RDC), du 01 mai 2017 au 15 janvier 2018, chez des enfants et adolescents VIH positif âgés de 0 à 19 ans. Les manifestations buccales ont été diagnostiquées selon les critères cliniques de Ramos-Gomez et al. La régression logistique, en analyse univariée et multivariée, exprimée en Odds ratio (OR) avec leur intervalle de confiance estimée à 95\%, a été exploitée pour rechercher les facteurs associés aux manifestations buccales.Résultats : Quatre cent vingt-deux patients étaient inscrits (219 filles et 203 garçons), l'âge médian était de 13 ans (IIQ : 10 ans-16 ans) ; 402 patients $(95,3 \%)$ recevaient les ARV; 359 patients $(85,1 \%)$ présentaient une ou plusieurs manifestations buccales. La carie dentaire était présente chez 161 patients $(38,9 \%)$. Le sexe féminin (ORa : 2,478; IC à 95 $\%[1,30-4,69]) ; \mathrm{p}=0,005)$ et l'hygiène buccale modérée et mauvaise (ORa : 4,54; IC à 95\% [1,74-11,835]; $\mathrm{p}=0,006)$ étaient les facteurs associés aux manifestations buccales.Conclusion : Les manifestations buccales chez les enfants et adolescents VIH positif sont encore très fréquentes dans notre milieu, à l'ère de la thérapie antirétrovirale. Cette situation indique la nécessité d'inclure la santé buccodentaire dans la prise en charge des enfants et adolescents VIH positif.

Mots-clés : manifestations buccales, VIH/SIDA, enfants et adolescents, Kinshasa, RDC

\section{SUMMARY}

Objectives: To show the extent of oral manifestations in HIV-positive children and adolescents, attending hospital in Kinshasa (DRC) and identify the associated factors. Methods: A cross-sectional analytical study, conducted in two pediatric hospitals in Kinshasa (DRC), from May 1, 2017 to January 15, 2018, in HIV-positive children and adolescents aged 0 to 19 years. Oral manifestations were diagnosed according to the clinical criteria of Ramos-Gomez et al. Logistic regression, in univariate and multivariate analysis, expressed in Odds Ratio (OR) with their estimated 95\% confidence interval, was used to search for factors associated with oral manifestations.Results: Four hundred twenty-two patients were enrolled (219 girls and 203 boys), the median age was 13 years (IQI: 10 years-16 years), 402 patients $(95.3 \%)$ received ARVs; 359 patients (85.1\%) had one or more oral manifestations. Dental caries was present in 161 patients $(38.9 \%)$. The female sex (ORa : 2,478; IC à $95 \%[1,30-4,69] ; \mathrm{p}=0,005)$ and moderate and poor oral hygiene (ORa : 4,54; IC à 95\% [1,74-11,835]; p $=0,006)$ were associated factors of oral manifestations.

Conclusions: Oral manifestations in HIV positive children and adolescents are still very common in our environment, in the era of antiretroviral therapy. This requires including oral health in the care of HIV-positive children and adolescents.

Keywords: oral manifestations, HIV/AIDS, children and adolescents, Kinshasa, DRC 


\section{INTRODUCTION}

L'infection à VIH est un problème majeur de santé publique dans le monde, particulièrement dans les pays à revenu faible ou intermédiaire ${ }^{[1]}$; avec environ 36,9 millions des personnes infectées en 2017, dont 1,8 millions d'enfants de moins de 15 ans. L'Afrique subsaharienne concentre la majorité des PVVIH avec environ 25,7 millions des PVVIH et près de $90 \%$ des enfants vivant avec le VIH. ${ }^{[1-3]}$ En République Démocratique du Congo (RDC), le nombre des PVVIH était de 381.187 en 2017 dont 48.000 enfants de moins de 15 ans. ${ }^{[4]}$ Chez les enfants, l'infection à VIH se transmet dans plus de $90 \%$ de cas de la mère à l'enfant. ${ }^{[5]}$ Cependant, grâce aux progrès dans le domaine de la prévention de la transmission de la mère à l'enfant, le nombre de nouvelles infections à VIH chez les enfants a diminué. [1, 2, 5] Le VIH affecte le système immunitaire de l'hôte, par destruction et altération fonctionnelle des cellules immunitaires. L'immunodépression installée prédispose l'enfant à la survenue des pathologies opportunistes parmi lesquelles figurent les manifestations buccales. ${ }^{[5]}$

Le TAR (traitement antirétroviral) a permis la diminution de la prévalence globale des manifestations buccales chez les adultes infectés par le VIH. Cependant, pour Yengopal et al. ${ }^{[6]}$ et Maeve et al. ${ }^{[7]}$, aucun changement n'a été observé chez les enfants infectés par le VIH. La prévalence des manifestations buccales survenant chez les enfants et les adolescents infectés par le VIH varie de 20 à $80 \%$. ${ }^{[6,8,9]}$ Ces manifestations buccales font partie des indicateurs cliniques précoces de l'infection à VIH, des marqueurs de l'immunodéficience et de l'efficacité du traitement antirétroviral, ainsi que de facteurs prédictifs de la progression de l'infection à VIH vers le SIDA. ${ }^{[10-12]}$ Ces manifestations buccales peuvent causer des douleurs et de l'inconfort, rendant difficile l'alimentation. ${ }^{[10]}$ Les enfants infectés par le VIH mal nourris peuvent présenter une détérioration clinique rapide de l'état général, aggravant ainsi la mortalité et la morbidité liées à l'infection à VIH. ${ }^{[5}$, 10, 13] En République Démocratique du Congo, aucune publication sur les manifestations buccales survenant au cours de l'infection à VIH chez les enfants et adolescents n'est disponible à Kinshasa (RDC). Ainsi, les objectifs de la présente étude était de montrer l'ampleur des manifestations buccales chez les enfants et adolescents infectés par le VIH à Kinshasa et d'en identifier les facteurs associés.

\section{METHODES}

Type et cadre d'étude : Nous avons conduit une étude transversale analytique, du 1er mai 2017 au 15 janvier 2018. Par choix raisonné, l'étude a été réalisée dans deux hôpitaux pédiatriques de Kinshasa: l'Hôpital Pédiatrique de Kalembe Lembe (HPKLL) et l'Hôpital Pédiatrique de Kimbondo (HPK).
Participants : $\mathrm{La}$ population d'étude était constituée d'enfants et adolescents infectés par le VIH, suivis régulièrement dans les unités des maladies infectieuses des hôpitaux enquêtés.

Critères d'éligibilité : Âge $0-19$ ans, être diagnostiqué positif pour le VIH de type 1 par PCR (enfant de moins de 18 mois) ou par sérologie (TDR, Western blot), le consentement éclairé écrit obtenu (parent ou le tuteur).

Critères de non éligibilité : enfant âgé de moins de 18 mois, diagnostiqué positif pour le VIH au seul test sérologique.

Taille de l'échantillon: Étant donné que la prévalence des manifestations buccales chez les enfants et adolescents infectés par VIH n'était pas connue, la taille de l'échantillon a été calculée selon la formule suivante $: n=\frac{t^{2} \cdot p(1-p)}{\AA^{2}}$ $n=\frac{(1,96)^{2} \cdot 0,5 \cdot 0,5}{(0,05)^{2}}=384,16=384$

En ajoutant le $10 \%$ de non - répondants possibles, nous avons inclus 422 patients. Un échantillon aléatoire et pondéré a été produit, selon le poids démographique des patients dans la fréquentation de chaque formation. La file active globale était de 1060 patients par an, 1000 patients pour l'HPKLL (soit un poids de 94,3\%) et 60 patients pour l'HPK (soit 5,7\%). Ainsi donc, sur 422 patients, nous avons inclus 398 patients à l'HPLL et 24 patients à l'HPK. Le recrutement des patients ne se faisait que les jours impairs ouvrables.

Variables étudiées : L'âge, le sexe, la consommation d'alcool et de tabac, la fréquence de consommation d'aliments et boissons dits sucrés, le brossage dentaire, la fréquence et les matériels de brossage dentaire, consultations dentaires antérieures, la durée de l'infection à $\mathrm{VIH}$, le stade clinique, la prise d'ARV, la durée du TAR, la prophylaxie au cotrimoxazole, la charge virale, le type des manifestations buccales, la présence des caries dentaires et l'état de l'hygiène buccale.

Pour la collecte des données, un questionnaire standardisé ${ }^{16}$ a permis de recueillir les informations sur les habitudes alimentaires, l'hygiène buccale et les habitudes des soins bucco - dentaires. Les données socio - démographiques (âge, sexe), les données cliniques et virologiques suivant ont été recueillies à partir des dossiers médicaux des patients (date de diagnostic de l'infection à VIH, stade clinique de l'OMS au moment de l'enquête tableau 1, adhérence au TAR, date d'adhérence au TAR, prophylaxie au cotrimoxazole et charge virale du VIH). Le stade clinique 4 a été considéré comme le stade clinique sévère. La charge virale $\geq 1000$ copies $/ \mathrm{ml} \mathrm{a}$ été considérée comme une charge virale élevée.

L'examen buccal a été réalisé à l'aide d'une sonde dentaire, d'un miroir dentaire, d'une abaisse langue et d'une lampe frontale à la recherche des lésions de la muqueuse buccale et des glandes salivaires principales. Les enquêteurs ont été calibrés sur les manifestations buccales associées à 
l'infection à VIH. Les manifestations buccales ont été diagnostiquées à partir des critères de diagnostic clinique décrit par le collaborative Workgroup on the Oral Manifestations of Pediatric HIV Infections. [14] L'hygiène buccale a été évaluée à l'aide de l'indice d'hygiène orale simplifiée (OHIS) de Greene et Vermillon ${ }^{[15]}$. L'OHIS comprend 2 indices, l'indice des débris (ID) et l'indice des tartres (IT). Les ID et IT comprennent chacune 4 scores ( 0 = absence des débris ou des tartres, 1 = débris ou tartres recouvrant le $1 / 3$ cervical de la face, $2=$ débris ou tartres recouvrant le $2 / 3$ cervical de la face, 3 = débris ou tartres recouvrent toute la face). Les ID et IT étaient prélevées sur les faces vestibulaires des dents $11,16,26,31$ et sur les faces linguales de la 36 et de la 46. Si la dent choisie était absente, elle était remplacée par la dent suivante. Si la dent suivante était absente, nous avions inscrit « dent absente ». L'ID et l'IT étaient obtenues en divisant la somme des scores de chaque indice sur le nombre des dents examinées. L'OHIS de chaque patient était obtenu en faisant la somme des ID et IT. Pour l'interprétation, lorsque l'OHIS était :

- égal à 0 : l'HB était considérée comme excellent ;

- $\quad$ situé entre 0,1 et 1,2: l'HB était considérée comme bonne ;

- $\quad$ situé entre 1,3 et 3,0: l'HB était considérée comme moyenne.

- $\quad$ situé entre 3,1 et 6,0: l'HB était considérée comme mauvaise.

Des séances de sensibilisation ont été organisées et chaque patient a reçu des informations sur la santé buccale et le maintien d'une bonne hygiène buccale.

Analyses statistiques : Les données étaient saisies à l'aide du logiciel Excel 2013. Les analyses ont été faites à l'aide du logiciel SPSS 20.0. Les variables quantitatives avec distribution gaussienne ont été présentées sous-forme de moyenne avec d'écarttype ou sous-forme de médiane avec les extrêmes et l'intervalle interquartile (IIQ), dans le cas contraire; les variables qualitatives sous forme de proportion. La fréquence des manifestations buccales a été estimée avec un intervalle de confiance (IC) de 95\%. Le test chi - carré et le test exact de Fisher ont permis de faire la comparaison entre les patients qui présentaient les manifestations buccales et ceux qui ne les présentaient pas. La recherche des facteurs associés aux manifestations buccales a été réalisée à l'aide de la régression logistique, en analyse uni et multivariée avec Odds ratio (OR) estimés à un IC de $95 \%$.

La valeur de $\mathrm{p}<0,05$ était considéré comme le seuil de significativité statistique.

\section{RESULTATS}

Caractéristiques générales des patients

Au total, 422 patients, âgés de 2 mois à 19 ans ont été examinés. L'âge médian (IIQ) était de 13 (10 -
16) ans; $219(51,9 \%)$ patients étaient de sexe féminin. $313(74,2 \%)$ patients consommaient régulièrement les aliments et boissons dits sucrés. $395(93,6 \%)$ patients se brossaient les dents. 273 $(69,1 \%)$ patients se brossaient les dents une fois par jour. 367 patients $(92,9 \%)$ utilisaient une brosse à dent avec dentifrice. $51(12,1 \%)$ avaient déjà consulté un service des soins dentaires. $16(3,9 \%)$ patients avaient un OHIS mauvais [Tableau II]. $211(50 \%)$ patients étaient au stade clinique 3 de l'infection à VIH. $349(84,5 \%)$ patients [ $\mathrm{n}=413$ ] avaient une durée de l'infection à VIH de plus de 2 ans. $402(95,3 \%)$ patients étaient sous TAR et parmi lesquels $316(78,6 \%)$ patients $(n=402)$ étaient sous TAR depuis plus de 2 ans. $318(75,4 \%)$ patients recevaient une prophylaxie au cotrimoxazole. $92(24,3 \%)$ patients avaient une charge virale $\geq 1000$ copies $/ \mathrm{ml}$. [Tableau III]

Prévalence des manifestations buccales

$\mathrm{Au}$ moment de l'étude, 359 patients $(85,1 \%)$ présentaient des manifestations buccales dont les plus couramment observées étaient l'infection à Molluscum contagiosum, la sécheresse buccale, l'érythème gingival linéaire et la candidose buccale [Tableau IV]. La carie dentaire avait une prévalence de $38,9 \%$ [Tableau V].

Facteurs associés aux manifestations de la muqueuse buccale

En analyse univariée, la présence des manifestations buccales étaient associées à l'âge de 5 à 9 ans (OR : 10,66; IC à 95\% [3,56 - 31,90], p $=0,000)$, au sexe féminin (OR : 1,78; IC à $95 \%$ $[1,03-3,00], \quad p=0,037)$, à un état moyen et mauvais de l'hygiène buccale reflétée par un OHIS $>1,3$ (OR : 5,42 ; IC à $95 \%[2,33$ - 12,61], p $=0,000)$ et à la durée de l'infection $>2$ ans (OR : $3,31 ; \mathrm{IC}$ à $95 \% \quad[1,66-6,60], \mathrm{p}=0,000)$ [Tableau VI].

En analyse multivariée, la présence des manifestations buccales étaient associées au sexe féminin (ORa: 2,478; IC à 95\% ([1,30 - 4,69]), p $=0,005]$ et à un état moyen et mauvais de l'hygiène buccale reflétée par un OHIS < 1,3 (ORa : 4,54; IC à 95\% [1,74-11,83]), p = 0,006) [Tableau VI].

\section{DISCUSSION}

Il s'agit d'une première étude sur les manifestations buccales survenant chez les enfants et les adolescents infectés par le VIH à Kinshasa (RDC). La population d'étude comprenait 422 patients âgés de 2 mois à 19 ans. La majorité des patients était sous TAR et $75,4 \%$ recevaient une prophylaxie au cotrimoxazole. Dans les pays à ressources limitées, la prévalence des manifestations buccales chez les enfants et les adolescents infectés par le VIH sous TAR varie selon les études, de plus de $70 \%$ (Rwenyonyi et al. ${ }^{[16]}$ Nabbanja et al. $\left.{ }^{[17]}\right)$ à près de $8 \%$ (Meless et al. ${ }^{[18]}$ ). Dans la présente étude, nous avons rapporté une prévalence élevée des manifestations buccales $(85,1 \%)$, quoique la majorité 
$(95,3 \%)$ des patients soit sous TAR. Cette prévalence élevée peut être due soit aux effets indésirables du TAR, étant donné que la majorité des patients $(78,6 \%)$ avaient une prise d'ARV de longue durée ( 2 ans), soit aux conséquences d'un échec thérapeutique dû à une mauvaise observance au TAR ou à une résistance virale indépendante à l'observance ou secondaire à une adhérence inadéquate. Au Mali, une étude similaire réalisée chez les patients adultes par Boubacar et al. ${ }^{[19]}$ a trouvé une prévalence élevée de $71 \%$. Pour Ramos-Gomez et al. ${ }^{[9]}$, l'utilisation des ARV peut entrainer des lésions des tissus mous ; et la prise à long terme des ARV prédispose aux maladies parodontales et augmente le risque de survenue des lésions néoplasiques bénignes associées au human papilloma virus (HPV). L'infection par Molluscum contagiosum, la sécheresse buccale et l'érythème gingival linéaire étaient les plus observées. Certains auteurs ont rapporté que le TAR a entraîné la diminution des manifestations buccales telles que la candidose buccale, et l'augmentation des manifestations bénignes telles que la sécheresse buccale, l'infection à l'HPV, les pathologies des glandes salivaires. ${ }^{[6,20,21]}$ La sécheresse buccale diminue la protection de la muqueuse buccale contre des nombreux agents microbiens, ce qui augmente les chances de survenue des manifestations buccales. La candidose buccale avait une prévalence de $14,9 \%$, la candidose pseudomembraneuse était la plus prédominante $(9,5 \%)$. Nos résultats se rapprochent de ceux d'Oyedeje et al. ${ }^{[22]}$ qui ont trouvé une prévalence de $17,2 \%$. La prévalence de la carie dentaire varie de 11 à $96,7 \%$ (Meless et al. ${ }^{[18]}$ ). Cette prévalence est élevée dans les pays à ressources limitées. Meless et al. ${ }^{[18],}$ Arrivé et al ${ }^{[23]}$ et Rwenyonyi et al. ${ }^{[16]}$ ont rapporté des fréquences élevées de $86 \%, 76 \%, 50,2 \%$ respectivement. Dans la présente étude, nous avons observé une prévalence de la carie dentaire de 38,9\%. Ces fréquences élevées de la carie dentaire dans nos pays est due à une alimentation riche en sucres, à des médicaments sous forme de sirop contenant des saccharoses, à la diminution de la protection de la cavité buccale due à la sécheresse buccale, à un accès limité au service des soins dentaires; dans notre étude, seuls $12,1 \%$ des patients avaient consulté un dentiste depuis leur naissance. Cette faible fréquentation des services bucco - dentaires peut s'expliquer par le fait que dans notre pays, les soins bucco - dentaires ne sont pris en charge ni par des organismes nationaux ou internationaux, ni par la sécurité sociale, mais aussi par l'absence des professionnels de santé bucco-dentaire dans la chaîne de prise de l'infection à VIH pédiatrique. Les manifestations buccales étaient associées au sexe féminin et à une hygiène buccale moyenne et à la mauvaise hygiène buccale. Meless et al. ${ }^{[18]}$ et Nabbanja et al. ${ }^{[17]}$ ont trouvé une association avec une mauvaise hygiène buccale. A l'absence d'une bonne hygiène buccale, la plaque dentaire s'accumule sur les surfaces dentaires, et les bactéries pathogènes présentes dans la cavité buccale peuvent attaquer la muqueuse buccale et le tissu dentaire ${ }^{[24]}$. Quant au sexe féminin, nous pensons que l'explication pourrait être d'ordre hormonal et psychique (pour les adolescents) mais aussi du fait que les femmes sont généralement plus affectées que les hommes par la maladie.

\section{CONCLUSION}

Les manifestations buccales chez les enfants et adolescents VIH positif sont encore très fréquentes dans notre milieu, à l'ère de la thérapie antirétrovirale. Cette situation nécessite d'inclure la santé bucco-dentaire dans la prise en charge des enfants et adolescents VIH positif.

Conflit d'intérêt : aucun

\section{REFERENCES}

1. VIH/sida Principaux faits. fiche d'information. OMS ; Juil 2018 [en ligne]. http : www.who.int/fr/news-room/fact-

sheets/detail/hiv-aids consulté en novembre 2018.

2. UNICEF, The AIDS epidemic continues to take a staggering toll, but progress is possible. Update Jan 2018 [en ligne]. http : www.data.unicef.org/ Consulté en mars 2018.

3. UNICEF, UNAIDS. Step Up the Pace: Towards an AIDS-free generation in West and Central Africa. Dakar: UNICEF Regional Office and UNAIDS Regional Support Team for West and Central Africa; 2017.

4. Plan d'accélération de la prise en charge du VIH/SIDA chez l'enfant et l'adolescent. PNLS ; Sept 2017

5. Réseau Africain pour les soins aux enfants affectés par le SIDA, éditeur. Manuel sur le sida pédiatrique en Afrique. $3^{\text {ème }}$ ed. Kampala : ANECCA ; 2017.

6. Yengopal V, Kolisa Y, Thekiso MD, Molete MP. The child and adolescent with HIV in resource poor countries. Oral Diseases 2016; 22: $25-34$.

7. Maeve M, Greenspan J, Challacombe SJ. Oral lesions in nfection with human immunodeficiency virus. Bulletin of the World Health Organisation 2005; 83: 700706.

8. Arrivé E, Meless D, Anaya-Saavedra G, Gallottini M, Pinzon LM, Ramirez-Amador V. 
The global burden of oral diseases in pediatric HIV - infected populations: a workshop report. Oral Diseases 2016; 22: 149 - 157.

9. Ramos-Gomez FJ, Folayan MO. Oral Health Considerations in HIV-Infected Children. Curr HIV/AIDS Rep 2013; 10: 283-293.

10. L'enjeu des maladies bucco-dentaires - un appel pour une action mondiale. L'Atlas de la santé bucco-dentaire. $2^{\text {nde }}$ éd. Genève : Fédération dentaire internationale (FDI) ; 2015.

11. Kakabadze T, Rukhadze N, Mshvidobadze K, Lomtadze M, Kandelaki G. Oral lesions in HIV - positive patients in Georgia. Georgian Medical News 2008; 165: 60-65.

12. Berberi A, Noujeim Z. AIDS: An Epidemiologic Study on correlation Between HIV - Related Oral Lesions and Plasma Levels of CD4, CD8 T Lymhocytes Counts and Ratio among 50 patients. Br J Med Res 2015; 6 (9): 859-866.

13. Juana FW. Recommandations pour la nutrition des enfants infectés par le VIH. OMS ; Avr 2011 [en ligne]. http : www.who.int/ Consulté en février 2017.

14. Ramos-Gomez FJ, Flaitz C, Catapano P, Murray P, Milnes AR, Dorenbaum A. Classification, diagnostic criteria, and treatment recommendations for orofacial manifestations in HIV-infected pediatric patients. J Clin Pediatr Dent 1999; 23 (2): 85 96

15. Wei SHY, Lang NP. Periodontal epidemiological indices for children and adolescents: II. Evaluation of oral hygiene; III. Clinical applications. Pediatr Dentistr 1982; 4 (1): 64-73.

16. Rwenyonyi CM, Kutesa A, Muwazi L, Okullo I, Kasangaki A, Kekitinwa A. Oral Manifestations in HIV/AIDS Infected Children. Eur J Dent 2011; 5 (3): 291-298.

17. Nabbanja J, Gitta S, Peterson S, Rwenyonyi CM. Orofacial manifestations in HIV positive children attending Mildway Clinic in Uganda. Odontology 2013; 101(1): 116-120.

18. Meless D, Ba B, Faye M, et al. Oral lesions among HIV - infected children on antiretroviral treatment in West Africa. Trop Med Int Health 2014; 19(3): 246-255.

19. Boubacar Ba, Issa Konate, Drissa Goïta, MBento Gilles Armel, Amady Coulibaly, Kadiatou Keita, Hapssa Koita, Alfousseyni Touré, Tiémoko Daniel Coulibaly, Mamadou Lamine Diombana, Sounkalo Dao. Affections bucco-dentaires associées à l'infection à VIH dans le service de maladies infectieuses du CHU de Point-G, Bamako. Med Buccale Chir Buccale 2017; 23: 5-11.

20. Oliscovicz NF, Pomarico L, de Araújo Castro GB, Souza IR. Effect of highly active antiretroviral therapy use on oral manifestations in pediatric patients infected with HIV. Indian J Dent Res 2015; 26 (2): 200-204.

21. Nittayanata W, Talungchit S, Jaruratanasirikul $S$, et al. Effects of long-term use of HAART on oral health status of HIV-infected subjects. J Oral Pathol Med 2010; 39(5): 397-406.

22. Oyedeje OA, Olarere OG, Oluwatiyin AE, Agelebe E. Oral and dental lesions in HIV infected Nigerian Children. Pan Afr Med J 2015; 20: 287-292.

23. Arrivé E, Kouakoussui A, Eboumou E, Akatia F, Rouet F, Msellati P. Carie dentaire, gingivite et VIH pédiatrique en Côte d'Ivoire. AOS 2010; 12 (252): 375-383.

24. Piette E, Mahy P. Stomatites bactériennes non specifiques. Encycl Méd Chir, Odontologie/Stomatologie 2005; 22-045-A10. Elsevier SAS. 
Tableau I : Classification en stades cliniques de l'infection pédiatrique à VIH selon l'OMS ${ }^{[5]}$

\begin{tabular}{|c|c|}
\hline Stade clinique & Signe clinique \\
\hline Stade 1 & $\begin{array}{l}\text { Asymptomatique } \\
\text { Adénopathie généralisée persistante }\end{array}$ \\
\hline Stade 2 & $\begin{array}{l}\text { Hépatosplénomégalie persistante inexpliquée } \\
\text { Infection extensive par un virus responsable de l'apparition de l'apparition de verrues (au } \\
\text { niveau de visage, couvrant plus de } 5 \% \text { de la surface du corps ou responsable de } \\
\text { modifications morphologiques disgracieuses) } \\
\text { Eruptions papulaires prurigineuses } \\
\text { Infections mycosiques des ongles } \\
\text { Erythème gingival linéaire } \\
\text { Infection extensive à virus du papillome humain ou molluscum contagiosum (couvrant } \\
\text { plus de } 5 \% \text { de la surface du corps ou au niveau de visage) } \\
\text { Ulcérations buccales récurrentes (plus de } 2 \text { épisodes en } 6 \text { mois) } \\
\text { Hypertrophie persistante et inexpliquée des parotides } \\
\text { Zona } \\
\text { Infections récurrentes ou chroniques des voies respiratoires hautes : otite moyenne, } \\
\text { otorrhée, sinusite, angine (au moins un épisode au cours des } 6 \text { derniers mois) }\end{array}$ \\
\hline Stade 3 & $\begin{array}{l}\text { Malnutrition modérée inexpliquée (-2 DS ou }-2 \mathrm{Z} \text { scores) ne répondant pas de façon } \\
\text { satisfaisante au traitement habituel } \\
\text { Diarrhée persistante inexpliquée ( } 14 \text { jours ou plus) } \\
\text { Fièvre persistante inexpliquée supérieure à } 37,5^{\circ} \mathrm{C} \text { (intermittente ou constante) pendant } \\
\text { plus de } 1 \text { mois } \\
\text { Candidose buccale persistante (après les } 6 \text { premières semaines de vie) } \\
\text { Leucoplasie chevelue de la bouche } \\
\text { Tuberculose ganglionnaire } \\
\text { Tuberculose pulmonaire } \\
\text { Pneumonies sévères récurrentes d'origine bactérienne présumée (épisode en cours et au } \\
\text { moins un autre épisode au cours des } 6 \text { derniers mois) } \\
\text { Gingivite/périodontite aigue nécrotique ulcérative } \\
\text { Pneumonie interstitielle lymphoïde symptomatique } \\
\text { Pathologie pulmonaire chronique associée au VIH, y compris la bronchiectasie } \\
\text { Anémie }\left(<8 \text { g/dl), neutropénie }\left(<500 \text { cellules } / \mathrm{mm}^{3}\right) \text { ou thrombopénie }\left(<50000 / \mathrm{mm}^{3}\right)\right. \\
\text { inexpliquée }\end{array}$ \\
\hline Stade 4 & $\begin{array}{l}\text { Emaciation sévère inexpliquée ou malnutrition sévère inexpliquée (-3 DS) ne répondant } \\
\text { pas de façon satisfaisante au traitement habituel } \\
\text { Pneumonie à Pneumocytis } \\
\text { Infections sévères récurrentes d'origine bactérienne présumée, par exemple : empyème, } \\
\text { pyomyosite, infection osseuse ou articulaire, méningite, mais ne comprenant pas les } \\
\text { pneumonies (épisode en cours et au moins un autre épisode au cours de } 6 \text { derniers mois) } \\
\text { Infection chronique par le virus de l'herpès oro-labiale, cutanée ou viscérale (quel que soit } \\
\text { le site) d'une durée supérieure à } 1 \text { mois) } \\
\text { Tuberculose extrapulmonaire } \\
\text { Sarcome de Kaposi } \\
\text { Candidose œsophagienne (ou candidose trachéale, bronchique ou pulmonaire) } \\
\text { Toxoplasmose du système nerveux central (après la période néonatale) } \\
\text { Encéphalopathie à VIH } \\
\text { Infection à cytomégalovirus (CMV), rétinite à CMV ou infection à CMV d'un autre } \\
\text { organe, débutant après l'âge de } 1 \text { mois } \\
\text { Cryptococcose extrapulmonaire, y compris la méningite } \\
\text { Toute mycose endémique disséminée (histoplasmose extrapulmonaire, coccidiomycose) } \\
\text { Cryptosporidiose ou Isosporidiose chronique avec diarrhée } \\
\text { Infection mycobactérienne non tuberculeuse disséminée } \\
\text { Fistule rectale acquise associée au VIH } \\
\text { Lymphome non Hodgikien cérébral ou à cellules B } \\
\text { Leucoencéphalopathie multifocale progressive } \\
\text { Cardiomyopathie ou néphropathie liée à VIH }\end{array}$ \\
\hline
\end{tabular}


Tableau II : Répartition des patients selon l'âge, le sexe, les habitudes alimentaires et l'hygiène buccale

\begin{tabular}{|c|c|c|}
\hline Caractéristique & $n=422$ & $\%$ \\
\hline \multicolumn{3}{|l|}{ Tranche d'âge } \\
\hline Médiane (IIQ, 95\%) & 13ans (10-16 ans) & \\
\hline Min - max & 2 mois -19 ans & \\
\hline 0 à 4 ans & 31 & 7,4 \\
\hline 5 à 9 ans & 66 & 15,6 \\
\hline 10 à 14 ans & 165 & 39,1 \\
\hline 15 à 19 ans & 160 & 37,9 \\
\hline \multicolumn{3}{|l|}{ Sexe } \\
\hline Masculin & 203 & 48,1 \\
\hline Féminin & 219 & 51,9 \\
\hline \multicolumn{3}{|l|}{ Consommation d'alcool } \\
\hline Oui & 16 & 3,8 \\
\hline Non & 406 & 96,2 \\
\hline \multicolumn{3}{|l|}{ Consommation de tabac } \\
\hline Oui & 1 & 0,2 \\
\hline Non & 421 & 99,8 \\
\hline Fréquence de consommation d'aliment et boissons sucrés & $\mathrm{n}=422$ & \\
\hline Régulièrement & 313 & 74,2 \\
\hline Souvent & 106 & 25,1 \\
\hline Rarement & 3 & 0,7 \\
\hline Consultation dentaire antérieure & $\mathrm{n}=422$ & \multirow{3}{*}{$\begin{array}{l}12,1 \\
87,9\end{array}$} \\
\hline Oui & 51 & \\
\hline Non & 371 & \\
\hline \multicolumn{3}{|l|}{ Brossage dentaire } \\
\hline Oui & 395 & 93,6 \\
\hline Non & 27 & 6,4 \\
\hline Matériel de brossage dentaire & $\mathrm{n}=395^{*}$ & \\
\hline Brosse à dent avec dentifrice & 367 & 92,9 \\
\hline Brosse à dent sans dentifrice & 20 & 5 \\
\hline Fibre végétale & 1 & 0,3 \\
\hline Autres* & 7 & 1,8 \\
\hline Consultation dentaire antérieure & $n=422$ & \\
\hline Oui & 51 & 12,1 \\
\hline Non & 371 & 87,9 \\
\hline OHIS & $\mathrm{n}=411 * *$ & \\
\hline Excellent & 50 & 12,2 \\
\hline Bon & 197 & 47,9 \\
\hline Moyen & 148 & 36 \\
\hline Mauvais & 16 & 3,9 \\
\hline
\end{tabular}

Autres* : braise, étoffe, doigt. OHIS : indice d'hygiène orale simplifié. * Nous n'avons tenu compte que des patients qui se brossaient les dents. **11 patients n'avaient pas encore des dents. 


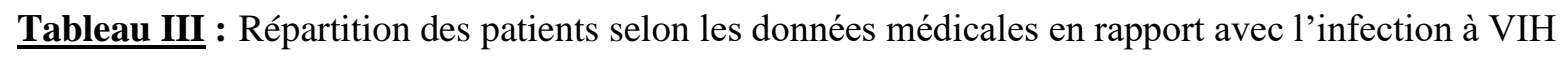

\begin{tabular}{|c|c|c|}
\hline Caractéristique & $\mathbf{n}$ & $\%$ \\
\hline Durée de l'infection à VIH & $n=413 *$ & \\
\hline Moins de 1 an & 49 & 11,9 \\
\hline 1 à 2 ans & 15 & 3,6 \\
\hline Plus de 2 ans & 349 & 84,5 \\
\hline Stade clinique & $n=422$ & \\
\hline Stade 1 & 67 & 15,9 \\
\hline Stade 2 & 115 & 27,3 \\
\hline Stade 3 & 211 & 50 \\
\hline Stade 4 & 29 & 6,9 \\
\hline TAR & $n=422$ & \\
\hline Oui & 402 & 95,3 \\
\hline Non & 20 & 4,7 \\
\hline Durée du TAR & $n=402 *$ & \\
\hline Moins de 1 an & 55 & 13,7 \\
\hline 1 à 2 ans & 31 & 7,7 \\
\hline Plus de 2 ans & 316 & 78,6 \\
\hline Prophylaxie cotrimoxazole & $n=422$ & \\
\hline Oui & 318 & 75,4 \\
\hline Non & 104 & 24,6 \\
\hline Charge virale & $\mathbf{n}=379 *$ & \\
\hline Médiane (IIQ : 95\%) & $<40$ copies/ml ( $<40-540$ copies $/ \mathrm{ml})$ & \\
\hline $\operatorname{Min}-\max$ & $<40-2402379$ copies/ml & \\
\hline$<1000$ & 287 & 75,7 \\
\hline$\geq 1000$ & 92 & 24,3 \\
\hline
\end{tabular}

VIH : virus de l'immunodéficience humaine. IIQ : intervalle interquartile. Min : minimum. max : maximum. < : Inférieure. $\geq$ : supérieure ou égale. $\mathrm{ml}:$ millilitre. *Certains dossiers de patient avaient des données manquantes. 


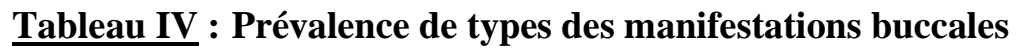

\begin{tabular}{lcc}
\hline Manifestations buccales & $\mathbf{n = 4 2 2}$ & $\mathbf{\%}$ \\
\hline Candidose buccale & $63^{*}$ & 14,9 \\
\multicolumn{1}{c}{ Pseudomembraneuse } & 40 & 9,5 \\
$\quad$ Erythémateuse & 24 & 5,7 \\
$\quad$ Chéilite angulaire & 8 & 1,9 \\
$\quad$ Gingivo-stomatite herpétique & $34 *$ & 8,1 \\
$\quad$ Herpès labiale récidivante & 1 & 0,2 \\
Infection à l'herpès simplex virus & 34 & 8,1 \\
Erythème gingivale linéaire & 136 & 32,2 \\
Tuméfaction des glandes salivaires & 48 & 11,4 \\
Ulcérations aphteuses récidivantes & 15 & 3,6 \\
Gingivite ulcéro-nécrotique & 42 & 9,9 \\
Parodontite ulcéro-nécrotique & 8 & 1,9 \\
Infections au human papilloma virus & 25 & 5,9 \\
Infection au molluscum contagiosum & 235 & 55,7 \\
Infection au virus du zona et de la varicelle & 1 & 0,2 \\
Sécheresse buccale & 205 & 48,6 \\
Sarcome de kaposi oral & 2 & 0,5
\end{tabular}

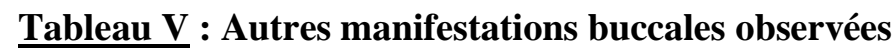

\begin{tabular}{lcc}
\hline Les autres manifestations buccales & $\mathbf{n}$ & $\mathbf{\%}$ \\
\hline Les atteintes de la muqueuse & $\mathbf{n = 4 2 2}$ & 1,7 \\
Ulcération atypique & 7 & 2,4 \\
Pétéchies & 10 & 3,3 \\
Chéilite exfoliatrice & 14 & 7,1 \\
Hyperpigmentation de la muqueuse buccale & 30 & 3,8 \\
Leucoplasie autre que la leucoplasie orale chevelue & 16 & 0,2 \\
Kyste lingual & 1 & $\mathbf{\%}$ \\
\hline Les atteintes dentaires & $\mathbf{n = 4 1 4}$ & 38,9 \\
Carie dentaire & 161 & 4,1 \\
Hypoplasie de l'émail & 17 & 11,4 \\
Persistance de dent temporaire & 47 & 8,8 \\
Dyschromie dentaire & 36 & 1,9 \\
Cellulite d'origine dentaire & 8 & 5,6 \\
Eruption dentaire retardée & 23 & \\
\hline
\end{tabular}


Tableau VI : Analyse des facteurs associés aux manifestations buccales en Analyse univariée et en analyse multivariée

\begin{tabular}{|c|c|c|c|c|c|c|c|c|}
\hline \multirow[b]{2}{*}{ Caractéristique } & \multicolumn{2}{|c|}{ Manifestations buccales } & \multirow[b]{2}{*}{$\boldsymbol{\beta}$} & \multirow[b]{2}{*}{ OR (IC 95\%) } & \multirow[b]{2}{*}{$p$} & \multirow[b]{2}{*}{$\bar{B}$} & \multirow[b]{2}{*}{ OR (IC 95\%) } & \multirow[b]{2}{*}{$p$} \\
\hline & $\begin{array}{c}\text { Absence } \\
\text { n(\%) }\end{array}$ & $\begin{array}{c}\text { Présence } \\
\mathbf{n}(\%)\end{array}$ & & & & & & \\
\hline Tranche d'âge & & & & & 0,00 & & & 0,161 \\
\hline 0 à 4 ans réf. & $16(25,4)$ & $15(4,2)$ & - & - & & - & - & \\
\hline 5 à 9 ans & $6(9,5)$ & $60(16,7)$ & 2,367 & $10,66(3,56-31,9)$ & & 1,25 & $3,51(0,78-15,76)$ & \\
\hline 10 à 14 ans & $25(39,7)$ & $140(39)$ & 1,787 & $5,97(2,62-13,60)$ & & 0,36 & $1,44(0,37-5,54)$ & \\
\hline 15 à 19 ans & $16(25,4)$ & $144(40,1)$ & 2,269 & $9,60(4,00-22,99)$ & & 0,89 & $2,45(0,61-9,76)$ & \\
\hline Sexe & & & & & $\mathbf{0 . 0 3}$ & & & $\mathbf{0 , 0 0 5}$ \\
\hline Masculin réf. & $38(60,3)$ & $165(46)$ & - & - & & - & - & \\
\hline Féminin & $25(39,7)$ & $194(54)$ & 0,581 & $1,78(1,03-3,00)$ & & 0,90 & $2,478(1,30-4,69)$ & \\
\hline $\begin{array}{l}\text { Consommation } \\
\text { sucréries }\end{array}$ & & & & & $\mathbf{0 , 3 9}$ & & & \\
\hline Rare/aucune réf. & $19(30,2)$ & $90(25,1)$ & & - & & & & \\
\hline Régulière & $44(68,8)$ & $269(74,9)$ & 0,225 & $1,29(0,71-2,32)$ & & & & \\
\hline OHIS* & & & & & $\mathbf{0 , 0 0}$ & & & 0,006 \\
\hline Excellent réf. & $15(27,3)$ & $35(9,8)$ & - & - & & - & - & \\
\hline bon & $28(50,9)$ & $169(47,5)$ & 0,950 & $2,58(1,25-5,34)$ & & 0,59 & $1,80(0,77-4,17)$ & \\
\hline Moyen/mauvais & $12(21,8)$ & $152(42,7)$ & 1,692 & $5,42(2,33-12,61)$ & & 1,51 & $4,54(1,74-11,835)$ & \\
\hline $\begin{array}{l}\text { Durée de } \\
\text { l'infection à VIH }\end{array}$ & & & & & $\mathbf{0 , 0 0}$ & & & $\mathbf{0 , 0 8 2}$ \\
\hline$<1$ an réf. & $15(27,3)$ & $34(9,7)$ & $-0,413$ & - & & $-1,25$ & - & \\
\hline 1 à 2 ans & $6(9,7)$ & $9(2,6)$ & 1,198 & $0,66(0,20-2,19)$ & & 0,28 & $0,28(0,06-1,30)$ & \\
\hline$>2$ ans & $41(66,1)$ & $308(87,7)$ & & $3,31(1,66-6,60)$ & & & $1,33(0,44-4,02)$ & \\
\hline Stade clinique & & & & & $\mathbf{0 , 1 0}$ & & & 0,659 \\
\hline Stade 1 réf. & $16(25,4 \%)$ & $51(14,2 \%)$ & - & - & & - & - & \\
\hline Stade 2 & $14(22,2 \%)$ & $101(28,1$ & 0,817 & $2,26(1,02-4,99)$ & & 1,16 & $1,18(0,43-3,20)$ & \\
\hline Stade 3 & $31(49,2 \%)$ & $\%)$ & 0,600 & $1,82(0,92-3,59)$ & & $-0,08$ & $0,91(0,36-2,31)$ & \\
\hline Stade 4 & $2(3,2 \%)$ & $\begin{array}{c}180(50,1 \\
\%) \\
27(7,5 \%)\end{array}$ & 1,443 & $4,23(0,90-19,80)$ & & 1,13 & $3,11(0,34-28,43)$ & \\
\hline Charge virale & & & & & 0.42 & & & \\
\hline$<1000$ réf. & $37(80,4)$ & $250(87,1)$ & & - & & & & \\
\hline$\geq 1000$ & $9(19,6)$ & $83(24,9)$ & 0,311 & $1,36(0,63-2,94)$ & & & & \\
\hline
\end{tabular}

* OHIS : indice d'hygiène orale simplifiée 